\title{
Correlations between cognitive impairment and brain-derived neurotrophic factor expression in the hippocampus of post-stroke depression rats
}

\author{
ZHAO-HUI ZHANG, LI-NA WU, JING-GUI SONG and WEN-QIANG LI \\ The Second Affiliated Hospital of Xinxiang Medical University, \\ Henan Key Laboratory of Biological Psychiatry, Xinxiang 453002, P.R. China
}

Received March 20, 2012; Accepted July 13, 2012

DOI: $10.3892 / \mathrm{mmr} .2012 .1009$

\begin{abstract}
The aim of this study was to investigate the correlation between brain-derived neurotrophic factor (BDNF) expression and cognitive impairment in post-stroke depression (PSD) rats and to explore the mechanism(s) involved in the process of cognitive impairment. A rat model of focal cerebral ischemia was established by occluding the middle cerebral artery (MCA). Rats were subjected to isolation-housing combined with chronic unexpected mild stress (CUMS) to establish a PSD rat model. The learning and memory abilities of the PSD rat model were evaluated by passive avoidance tests. Real-time PCR and immunohistochemical methods were used to detect changes in BDNF mRNA and protein expression in the hippocampus. Passive avoidance defects were revealed in the PSD and depression groups. Passive avoidance defects were more evident in the PSD group compared with the depression group and the difference was statistically significant $(\mathrm{P}<0.05)$. BDNF expression in the hippocampus was significantly lower in the PSD and depression groups compared with that in the normal control group $(\mathrm{P}<0.01)$. No significant difference in BDNF expression was identified between the normal control and stroke groups $(\mathrm{P}>0.05)$ or between the PSD and the depression groups $(\mathrm{P}>0.05)$. The decrease in BDNF expression in the hippocampus of PSD rats may aggravate cognitive impairment, however, the degree of cognitive impairment cannot be reflected by the expression levels of BDNF in the hippocampus.
\end{abstract}

Correspondence to: Professor Jing-Gui Song, The Second Affiliated Hospital of Xinxiang Medical University, Henan Key Laboratory of Biological Psychiatry, No. 388 Jianshe Middle Road, Xinxiang 453002, P.R. China

E-mail: songjg62@126.com

Key words: post-stroke depression, hippocampus, brain-derived neurotrophic factor, cognitive function

\section{Introduction}

Previous studies showed that the incidence of post-stroke depression (PSD) is greater than $25-54 \%$ (1). It has been reported that PSD delayed neurological injury recovery time, and was often associated with cognitive dysfunction (2). Depression and cognitive impairment in PSD patients influence each other, seriously affecting the patient's rehabilitation and quality of life and increasing the burden on family and society (3). With the incidence of PSD rising year by year, studies concerning cognitive impairment in PSD are attracting more attention. Brain-derived neurotrophic factor (BDNF) is a member of the nerve growth factor family and is expressed in the adult mammalian central and peripheral nervous system, particularly in the hippocampus and cortex. Clinical studies have shown that serum BDNF levels and hippocampal volume reductions in elderly individuals are closely correlated with memory loss. Further analysis has suggested that lower levels of BDNF cause hippocampal volumes to decrease, mediating agerelated spatial memory deficits $(4,5)$. BDNF is correlated with neurodegenerative diseases including Alzheimer's disease (6) and also has a strong correlation with other mental illnesses, including depression (7). Levels of BDNF are decreased in PSD patients compared with that in normal serum (8). Serum BDNF levels of ischemic stroke patients after admission to hospital for one day may be used to predict the subsequent risk of depression after stroke, with a decrease indicating the likelihood of development (9). Serum BDNF levels are closely correlated with emotional and cognitive impairment and other symptoms (10). In this study, the correlation between changes of BDNF expression in the hippocampus of PSD rats and cognitive impairment was analyzed and the mechanism of BDNF in the process of cognitive impairment in PSD was investigated.

\section{Materials and methods}

Experimental animals. A total of 60 clean grade and healthy Sprague-Dawley (SD) adult male rats (provided by the Experimental Animal Center of Zhengzhou University) were maintained with free access to food and water (excluding fasting and water deprivation prior to surgery and during stress). The raising conditions were at room temperature $\left(\sim 20-22^{\circ} \mathrm{C}\right)$, at a 
relative humidity of 60-70\% and light-dark cycles of $12 \mathrm{~h}$. Rats weighing 250-310 $\mathrm{g}$ were used in the experiments. This study was carried out in strict accordance with the recommendations in the Guide for the Care and Use of Laboratory Animals of the National Institutes of Health. The animal use protocol was reviewed and approved by the Henan Key Lab of Biological Psychiatry of Xinxiang Medical University, China.

Animal groups. The sucrose solution consumption test was conducted during pre-raising. After two weeks, the open-field test (OFT) was used for behavior scoring and sucrose solution consumption and baselines were measured. Forty-eight rats with uniform behavior were selected and randomly divided into four groups as follows: normal, stroke, depression and PSD groups (when rats died after surgery, the groups were randomly re-made up to six rats). In the normal group, shamoperated animals underwent the same surgical procedure, except the suture was not introduced into the external carotid artery. In the depression group, the rats were subjected to isolation-housing in combination with chronic unexpected mild stress (CUMS) to set up a depression model. In the stroke group, a middle cerebral artery occlusion (MCAO) model was established using the thread embolism method. Rats with postoperative neurological scores $\geq 1$ and $<4$ were selected according to the Longa 5-point scale scoring system. In the PSD group, rats in the MCAO model were subjected to isolation-housing 7 days following surgery.

Animal models of ischemic stroke. The rats were fasted for $12 \mathrm{~h}$ before surgery. Methods were improved for further use according to Koizumi's method. Anesthesia was induced by the injection of $0.3 \%$ pentobarbital sodium $(1 \mathrm{ml} / 100 \mathrm{~g}$ body weight) and repeated as required. Rats were fixed onto a wooden operating table, and the neck skin was prepared for surgery by regular disinfection. The subcutaneous tissues were dissected through a ventral midline incision on the left side of neck to avoid damaging the submandibular gland, lymph nodes and other structures, and the sternohyoid and sternocleidomastoid muscles were exposed and isolated. Left common carotid artery (CCA) trunks, CCA bifurcation, external main carotid artery (ECA) and internal carotid artery (ICA) were exposed and isolated under the operating microscope. The CCA trunks and proximal end of the ECA were coagulated. The suture was tied loosely around the distal CCA and ICA trunk and a curved microvascular clip was placed across the distal ICA end with temporary occlusion of the distal arteriole ICA. A small hole was cut 3-4 $\mathrm{mm}$ from the CCA branches and the microvascular clip was removed. The suture was then gently advanced into the ICA. The arc-shaped suture was inserted outward and upward to prevent incorrect insertion into the pterygopalatine artery (PPA). When a suture was inserted only $10 \mathrm{~mm}$, resistance suggested that the suture was mistakenly inserted into the PPA. Therefore, the suture needed to be inserted again after adjusting direction, until more resistance was felt. When a suture was inserted $17-20 \mathrm{~mm}$ deep (distal to the CCA bifurcation), the suture accurately reached the initial segment of the middle cerebral artery (MCA). The intraluminal suture blocked the blood flow into the MCA to cause focal cerebral ischemia. The suture in the distal CCA and ICA trunk was coagulated to prevent suture loss and the extra suture was cut.
The operating site was cleaned with sterile cotton balls and then sutured. Sodium penicillin (40,000 units) was injected intraperitoneally to prevent postoperative infection. The rat's head was directly exposed to a $60 \mathrm{~W}$ incandescent lamp at a height of $37 \mathrm{~cm}$, maintaining the air flow and reducing fluctuations of body and brain temperature, until revival. Ischemic neurological deficit was assessed when the rats were awake.

Establishment of rat models of PSD. The rats with focal cerebral ischemia prepared according to the abovementioned steps were subjected to isolation-housing. After 7 days of feeding, CUMS was conducted for 21 days, including seven stimulation methods including fasting for $24 \mathrm{~h}$, water deprivation for $24 \mathrm{~h}$, noise stimulation, forced swimming, foot shocking, tail clamping and behavioral restrictions for $120 \mathrm{~min}$. Each stimulation method was used three times and randomly distributed into the three stress cycles (21 days). The methods involved: i) fasting: a total of 24-h fasting from 7:00 am to 7:00 am the next day; ii) water deprivation: a total of $24 \mathrm{~h}$ water deprivation from 7:00 am to 7:00 am the next day; iii) noise stimulus: the rats were placed in a closed room with $1,500 \mathrm{~Hz}, 95 \mathrm{~dB}$ noise for $1 \mathrm{~h}$ every day; iv) forced swimming: the rats were placed in a bucket containing ice water $\left(40 \mathrm{~cm}\right.$ depth) at $4^{\circ} \mathrm{C}$ for 1-3 times every day (5-15 $\mathrm{min} / \mathrm{time})$; v) foot shocking: a total of 20 foot shocks (voltage $60 \mathrm{~V}$, current $1 \mathrm{~mA}$ ) were conducted for $10 \mathrm{sec}$ with a 1-min interval; vi) tail clamping: the rats were placed in the behavior limiter with exposed tails. A large oval clamp was placed over the tail near the lateral third of the body for $1 \mathrm{~min}$ and then the tail was placed into the cage (without using too much force); vii) behavioral restrictions for $120 \mathrm{~min}$ : the rats were placed in a rat behavior limiter, $2 \mathrm{~h} /$ time, 1 time/day.

General condition. The mental state of the rats, eating and drinking habits, stools, purulent neck incisions and the number of dead rats were observed daily. The time of death was recorded and the cause of death was determined by autopsy. Body weight was retested once a week.

The functional neurological deficits of focal cerebral ischemia rats were scored according to the Longa 5-grade standards: 0 point for no neurological deficit; 1 for a mild focal neurological deficit (failure to extend left forepaw fully); 2 for a moderate focal neurological deficit (circling to the left); 3 for a severe focal deficit (falling to the left); 4 for the inability to walk spontaneously and unconsciousness.

Sugar solution consumption. Rats were deprived of water for $24 \mathrm{~h}$ prior to the test, and then $1 \%$ sucrose solution was provided for $1 \mathrm{~h}$. Consumption was calculated as the amount of sucrose solution consumed $(\mathrm{g} / 100 \mathrm{~g})=$ the amount consumed (g) for $1 \mathrm{~h} /$ animal body weight $(\mathrm{g})$ x 100 .

Open field test analysis. The open box used was $100 \times 100 \times 50 \mathrm{~cm}$ in size with opaque black walls. On the bottom there were 25 equal-area grids divided by white lines. The observed rats were placed in a single central grid in the box and their behavior was recorded for $3 \mathrm{~min}$, including horizontal crossing (times of stepping into the neighboring squares by three claws) and vertical frequency (times forelimbs were elevated at least $1 \mathrm{~cm}$ from the ground or were clung to the walls). The open box 
was thoroughly cleaned before the next rat was observed. The time of behavioral assessment was fixed at 8:00-12:00 am. The assessment was conducted in a quiet room by two observers. The original behavior data of rats were the average of the data from the two observers.

Passive avoidance test. As described previously (2), the training test was conducted first. The animals were placed into the white compartment and the middle door was closed. The rats were given a few seconds to become familiar with the environment and then the door was opened. The time taken to enter the dark compartment was recorded, and was designated as incubation period 1 . If the animal did not enter the dark compartment in a pre-set time, the test was terminated and the animals were eliminated. When the animal entered the dark compartment, the door was closed. After a brief electric shock, the rats were returned to the cage and the compartment was thoroughly cleaned. The animals were put into the white compartment $24 \mathrm{~h}$ after the training test for a few minutes to become familiar with the environment. The time starting from the middle door opening to the animals entering the dark compartment was recorded as incubation period 2 .

Immunohistochemistry. The right half of the brain tissue was fixed, embedded in paraffin and sliced and the sections were rehydrated using the normal method. The sections were incubated with $3 \% \mathrm{H}_{2} \mathrm{O}_{2}$ for 10 min to eliminate peroxidase activity and were then washed three times for $2 \mathrm{~min}$. Antigen was retrieved in sodium citrate by microwave-heating for 15 min and was then cooled. After adding blocking solution

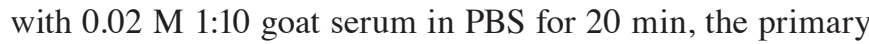
antibody was added overnight at $4^{\circ} \mathrm{C}$. After rinsing three times for 2 min with PBS, biotinylated secondary antibodies were added and incubated at $20-37^{\circ} \mathrm{C}$ for $20 \mathrm{~min}$. After washing three times for 2 min with $\mathrm{PBS}, \mathrm{SABC}$ working solution was added and incubated at $20-37^{\circ} \mathrm{C}$ for $20 \mathrm{~min}$. After washing four times for $5 \mathrm{~min}$ with $\mathrm{PBS}$, the sections were colored with $\mathrm{DAB}$ reagent at room temperature.

Real-time quantitative PCR. The left brain tissue was frozen in liquid nitrogen and used for total RNA extraction and reverse transcription. In this study, the GAPDH gene (forward: 5'-ACCACAGTCCATGCCATCAC-3' and reverse: 5'-TCC ACCACCCTGTTGCTGTA-3') was the internal control standard. Primers of the rat BDNF gene (forward: 5'-CACACACA GCGCTCCTTA-3' and reverse: 5'-AGTGGTGGTCT GAGGTTGG-3') were synthesized by Invitrogen Corporation (Carlsbad, CA, USA). The cDNA obtained by reverse transcription was used for fluorescence quantitative PCR. The reactions were incubated at $95^{\circ} \mathrm{C}$ for $120 \mathrm{sec}$, followed by 40 cycles of $95^{\circ} \mathrm{C}$ for $20 \mathrm{sec}, 57^{\circ} \mathrm{C}$ for $45 \mathrm{sec}$ and $72^{\circ} \mathrm{C}$ for $60 \mathrm{sec}$.

Statistical analysis. Data were analyzed with SPSS 12.0 statistical software and all measurement data were expressed as the mean values \pm standard deviation. The data were firstly checked using the normal distribution and homogeneity of variance test, and then analyzed according with the normal distribution and homogeneity of variance between the two groups analyzed using an independent sample t-test. Repeated measures design analysis of variance (ANOVA) was used to analyze the mean in the multigroup. $\mathrm{P}<0.05$ was considered to indicate statistically significant differences.

\section{Results}

Changes of body weight. One day after surgery, body weight was reduced in the stroke and PSD groups compared with the normal group and the difference was statistically significant $(\mathrm{P}<0.01)$. No significant difference was identified in body weight between the depression and normal groups $(\mathrm{P}>0.05)$. During days 7-28 after surgery, the body weight of the rats in each group increased. The increased ranges in the PSD and depression groups were lower than those in the normal and stroke groups. At 7 days after surgery, body weight was lower in the stroke and PSD groups than that in the normal group and the difference was statistically significant $(\mathrm{P}<0.05)$. No significant difference was identified in body weight between the depression and normal groups $(\mathrm{P}>0.05)$. At 28 days after surgery, body weight was lower in the stroke, depression and PSD groups than in the normal group and the differences were statistically significant $(\mathrm{P}<0.05)$.

Changes of sucrose solution consumption. No significant difference was identified in the consumption of sucrose solution between the normal and other groups at 7 days before and after surgery $(\mathrm{P}>0.05)$. The consumption of sucrose solution in the depression and PSD groups was less than that in the normal group 28 days after surgery and the difference was statistically significant $(\mathrm{P}<0.05)$. No significant difference was identified between the stroke and normal groups $(\mathrm{P}>0.05)$. No significant difference was identified between the depression and PSD groups $(\mathrm{P}>0.05)$.

Changes of rat movement. No significant difference was identified in the times of horizontal movement between the normal and other groups at 7 days before and after surgery $(\mathrm{P}>0.05)$. At 28 days after surgery, the times of horizontal movement in the depression and PSD groups were less than those in the normal group and the difference was statistically significant $(\mathrm{P}<0.05)$. No significant difference was identified between the stroke and normal groups $(\mathrm{P}>0.05)$. The times of horizontal movement of rats in the PSD group were less than those in the depression group, and the difference was statistically significant $(\mathrm{P}<0.05)$.

At 7 days before and after surgery, no significant difference was identified in the times of vertical movement between the normal and other groups ( $\mathrm{P}>0.05)$. At 28 days after surgery, the times of vertical movement in the depression and PSD groups were less than those in the normal group and the difference was statistically significant $(\mathrm{P}<0.05)$. No significant difference was identified between the stroke and normal groups $(\mathrm{P}>0.05)$ or the stroke and PSD groups $(\mathrm{P}>0.05)$.

Passive avoidance test. No significant difference was identified in the time for rats to enter the dark compartment between the normal and other groups in the training test $(\mathrm{P}>0.05)$. At $24 \mathrm{~h}$ after the training test, the time for rats to enter the dark compartment was shorter in the depression and PSD groups than that in the normal group and the difference was statisti- 
Table I. Results of the passive avoidance test in rats.

\begin{tabular}{lccc}
\hline Groups & Cases $(\mathrm{n})$ & $\begin{array}{c}\text { Training test } \\
(\mathrm{sec})\end{array}$ & $\begin{array}{c}24 \mathrm{~h} \text { after training test } \\
(\mathrm{sec})\end{array}$ \\
\hline Normal & 6 & $15.50 \pm 2.68$ & $208.32 \pm 19.43$ \\
Stroke & 6 & $14.63 \pm 4.15$ & $198.52 \pm 12.80$ \\
Depression & 6 & $18.70 \pm 5.66$ & $32.20 \pm 7.18^{\mathrm{a}}$ \\
PSD & 6 & $19.86 \pm 2.40$ & $17.24 \pm 1.56^{\mathrm{a}, \mathrm{b}}$ \\
F & & 2.304 & 431.128 \\
P & & 0.108 & 0.000 \\
\hline
\end{tabular}

${ }^{\text {ap }}<0.05$ compared with the normal group; ${ }^{\mathrm{b}} \mathrm{P}<0.05$ compared with the depression group. BDNF, brain-derived neurotrophic factor; PSD, post-stroke depression.

Table II. Real-time PCR results of BDNF mRNA.

\begin{tabular}{lcc}
\hline Groups & Cases $(\mathrm{n})$ & BDNF mRNA \\
\hline Normal & 6 & $1.15 \pm 0.07$ \\
Stroke & 6 & $1.11 \pm 0.04$ \\
Depression & 6 & $0.87 \pm 0.10^{\mathrm{a}}$ \\
PSD & 6 & $0.85 \pm 0.10^{\mathrm{a}}$ \\
F & & 22.019 \\
P & & 0.000 \\
\hline
\end{tabular}

${ }^{\mathrm{a}} \mathrm{P}<0.05$ compared to the normal group. BDNF, brain-derived neurotrophic factor; PSD, post-stroke depression.

cally significant $(\mathrm{P}<0.05)$. The time for rats to enter the dark compartment was shorter in the PSD than that in the depression group and the difference was statistically significant $(\mathrm{P}<0.05$; Table I). The time for rats to enter the dark compartment $24 \mathrm{~h}$ after the training test was prolonged in the normal and stroke groups and the time did not change in the depression and PSD groups.

Immunohistochemistry. The average optical density of hippocampal BDNF with positive staining was lower in the depression and PSD groups than that in the normal group and the difference was statistically significant $(\mathrm{P}<0.05)$. No significant differences were identified between the stroke and control groups $(\mathrm{P}>0.05)$ or the depression and PSD groups $(\mathrm{P}>0.05)$.

Real-time PCR results of BDNF. The expression of BDNF mRNA in rat hippocampus was lower in the depression and PSD groups than that in the normal group and the difference was statistically significant $(\mathrm{P}<0.05)$. No significant differences were identified between the stroke and control groups $(\mathrm{P}>0.05)$ or the depression and PSD groups ( $\mathrm{P}>0.05$; Table II).

\section{Discussion}

Findings of this study have shown that the learning ability and memory of rats were decreased in the PSD and depression groups, with this decrease being more significant in the PSD than the depression group. Compared with the normal group, the hippocampal BDNF expression decreased in the PSD and depression groups and there was no significant difference between these groups. Therefore, BDNF may be involved in the biological process of learning and memory, but it does not reflect the degree of cognitive impairment. The functions of BDNF in learning and memory may be to promote the survival rate and growth of cholinergic, GABA, dopaminergic, motor, sensory, cerebellar granule $(11,12)$ and basal forebrain cholinergic neurons, which are a significant component of neural circuits in learning and memory. BDNF enhances the density of synaptic terminals, promotes the growth of axons and dendrites, promotes the transmission and plasticity of synaptic terminals (13-15) and is involved in the long-term potentiation and plasticity of learning and memory $(16,17)$. Studies show that there is inherent contact between the BDNF-Trk B signaling pathway and the NMDA receptor, which is crucial in the spatial memory of the hippocampus (18). BDNF is the toxic antagonist to excitatory amino acids $(19,20)$.

A number of studies have shown that the expression of BDNF is not consistent with ischemic time. This may be due to the extent and type of cerebral ischemia, the state of experimental animals and the various detection methods of BDNF. It is generally considered that the expression of BDNF mRNA and its protein in patients with cerebral ischemia only rises transiently, and is gradually reduced over time. This study shows that there was no significant difference in the main behavioral changes, learning and memory and the expression of hippocampal BDNF between rats in the stroke and normal groups. The reason may be that BDNF in the stroke rats had first increased, decreased and then returned to normal during the experiment. Distinct from humans, the anterior cerebral artery in the rat brain combines with the artery of the same name on the contralateral side to form the anterior cerebral artery, which enters the anterior cerebral fissure, reaches the top and then joins the branch of the posterior cerebral artery. Longa et al (21) believed that blood in the rat thalamus and basal ganglia was supplied not only by the aforementioned arterial branches but also by the blood flow in the pterygopalatine artery, which was not occluded in this experiment. The above points prove that the compensatory ability of cerebral blood flow in rats is stronger than that in humans, suggesting that artery occlusion stroke on one side of the brain in rats generally does not cause depression or cognitive dysfunction in the absence of adverse external circumstances or stimulation and tends to restore back to normal.

BDNF may be involved in the entire pathological process during the development of cerebral ischemia to PSD and then cognitive impairment. Numerous studies (22) have shown that a series of cascade reactions occur in the early stages of cerebral ischemia including, excitatory amino acid release, neuronal depolarization and $\mathrm{Ca}^{2+}$ influx, these cascade reactions cause an increase in BDNF expression. Endogenous BDNF achieves its biological effects and protects ischemic neurons in a variety of ways. It plays a key role in the process of nerve cell survival, growth and recovery. When humans or animals are under a variety of adverse stress states at the same time with prolonged ischemia, levels of glucocorticoid are elevated over a long period of time, leading to an impaired 
receptor sensitivity and a reduced density of glucocorticoid and disinhibition of the hippocampal neuronal axis, resulting in a persistent increase of cortisol levels. Long-term high levels of glucocorticoids reduce the size of the hippocampus and decrease synaptic density in the hippocampal region (23). Decreases in mRNA and protein levels of BDNF in the rat hippocampus also occur (24) as well as a reduction of nerve-transmitter release mediated by $\operatorname{BDNF}(25)$, resulting in the decline of learning, memory and other cognitive functions.

\section{References}

1. Haq SU, Symeon C, Agius M and Brady R: Screening for depression in post stroke patients. Psychiatr Danub 22: 33-35, 2010.

2. Mihara T, Mihara K, Yarimizu J, et al: Pharmacological characterization of a novel, potent adenosine $\mathrm{A} 1$ and $\mathrm{A} 2$ receptor dual antagonist, 5-[5-amino-3-(4-fluorophenyl)pyrazin-2-yl]-1-isopropylpyridine-2(1H)-one(ASP5854), in models of Parkinson's disease and cognition. J Pharmacol Exp Ther 323: 708-719, 2007.

3. Barba R, Martínez-Espinosa S, Rodríguez-García E, Pondal M, Vivancos J and Del Ser T: Post-stroke dementia: clinical features and risk factors. Stroke 31: 1494-1501, 2000.

4. Erickson KI, Prakash RS, Voss MW, et al: Brain-derived neurotrophic factor is associated with age-related decline in hippocampal volume. J Neurosci 30: 5368-5375, 2010.

5. Li G, Peskind ER, Millard SP, et al: Cerebrospinal fluid concentration of brain-derived neurotrophic factor and cognitive function in non-demented subjects. PLoS One 4: e5424, 2009.

6. O'Bryant SE, Hobson VL, Hall JR, et al: Serum brain-derived neurotrophic factor levels are specifically associated with memory performance among Alzheimer's disease cases. Dement Geriatr Cogn Disord 31: 31-6, 2011.

7. von Bohlen und Halbach O: Involvement of BDNF in agedependent alterations in the hippocampus. Front Aging Neurosci 13: 2,2010

8. Zhou Z, Lu T, Xu G, et al: Decreased serum brain-derived neurotrophic factor (BDNF) is associated with post-stroke depression but not with BDNF gene Val66Met polymorphism. Clin Chem Lab Med 49: 185-189, 2011.

9. Yang L, Zhang Z, Sun D, et al: Low serum BDNF may indicate the development of PSD in patients with acute ischemic stroke. Int J Geriatr Psychiatry 26: 495-502, 2011.

10. Teixeira AL, Barbosa IG, Diniz BS and Kummer A: Circulating levels of brain-derived neurotrophic factor: correlation with mood, cognition and motor function. Biomark Med 4: 871-887, 2010.

11. Murer M, Yan Q and Raisman-Vozari R: Brain-derived neurotrophic factor in the control human brain, and in Alzheimer's disease and Parkinson's disease. Prog Neurobiol 63: 71-124, 2001.
12. McAllister AK: Neurotrophins and neuronal differentiation in the central nervous system. Cell Mol Life Sci 58: 1054-1060, 2001.

13. Mertz K, Koscheck T and Schilling K: Brain-derived neurotrophic factor modulates dendritic morphology of cerebellar basket and stellate cells: an in vitro study. Neuroscience 97: 303-310, 2000.

14. Ohira K and Hayashi M: A new aspect of the TrkB signaling pathway in neural plasticity. Curr Neuropharmacol 7: 276-285, 2009.

15. Yoshii A and Constantine-Paton M: Post-synaptic BDNF-TrkB signaling in synapse maturation, plasticity and disease. Dev Neurobiol 70: 304-322, 2010.

16. Figurov A, Pozzo-Miller LD, Olafsson P, Wang T and Lu B: Regulation of synaptic responses to high frequency stimulation and LTP by neurotrophins in the hippocampus. Nature 381 : 706-709, 1996.

17. Cunha C, Brambilla R and Thomas KL: A simple role for BDNF in learning and memory? Front Mol Neurosci 3: 1, 2010.

18. Angelucci F, Mathé AA and Aloe L: Neurotrophic factors and CNS disorders: findings in rodent models of depression and schizophrenia. Prog Brain Res 146: 151-165, 2004.

19. Almeida RD, Manadas BJ, Melo CV, et al: Neuroprotection by BDNF against glutamate-induced apoptotic cell death is mediated by ERK and PI3-kinase pathways. Cell Death Differ 12: 1329-1343, 2005

20. Harper MM, Adamson L, Blits B, Bunge MB, Grozdanic SD and Sakaguchi DS: Brain-derived neurotrophic factor released from engineered mesenchymal stem cells attenuates glutamate- and hydrogen peroxide-mediated death of staurosporine-differentiated RGC-5 cells. Exp Eye Res 89: 538-548, 2009.

21. Longa EZ, Weinstein PR, Carlson S and Cummins R: Reversible middle cerebral artery occlusion without craniectomy in rats. Stroke 20: 84-91, 1989.

22. Xu J, Zhang QG, Li C and Zhang GY: Subtoxic N-methyl-Daspartate delayed neuronal death in ischemic brain injury through TrkB receptor- and calmodulin-mediated PI-3K/Akt pathway activation. Hippocampus 14:525-537, 2007.

23. Tata DA, Marciano VA and Anderson BJ: Synapse loss from chronically elevated glucocorticoids: relationship to neuropil volume and cell number in hippocampal area CA3. J Comp Neurol 498: 363-374, 2006.

24. Jacobsen JP and Mørk A: Chronic corticosterone decreases brain-derived neurotrophic factor (BDNF) mRNA and protein in the hippocampus, but not in the frontal cortex, of the rat. Brain Res 110: 221-225, 2006.

25. Numakawa T, Kumamaru E, Adachi N, Yagasaki Y, Izumi A and Kunugi H: Glucocorticoid receptor interaction with TrkB promotes BDNF-triggered PLC-gamma signaling for glutamate release via a glutamate transporter. Proc Natl Acad Sci USA 106: 647-652, 2009 